\title{
BMJ Open Risk of venous thromboembolism in patients undergoing gastric cancer surgery: protocol for a systematic review and meta-analysis
}

\author{
Dengfeng Wang, ${ }^{1,2}$ Yang Yu (D) , 1,3 Pengxian Tao, ${ }^{1,3}$ Dan Wang, ${ }^{1,3}$ Yajing Chen, ${ }^{1,3}$
} Hao Chen ${ }^{1}$

To cite: Wang D, Yu Y, Tao $\mathrm{P}$, et al. Risk of venous thromboembolism in patients undergoing gastric cancer surgery: protocol for a systematic review and meta-analysis. BMJ Open 2020;10:e033267. doi:10.1136/ bmjopen-2019-033267

- Prepublication history for this paper is available online. To view these files, please visit the journal online (http://dx.doi. org/10.1136/bmjopen-2019033267).

DW and YY contributed equally.

DW and YY are joint first authors.

Received 28 July 2019 Revised 29 0ctober 2019 Accepted 31 0ctober 2019

Check for updates

(C) Author(s) (or their employer(s)) 2020. Re-use permitted under CC BY-NC. No commercial re-use. See rights and permissions. Published by BMJ.

${ }^{1}$ Department of Tumor Surgery, Lanzhou University Second Hospital, Lanzhou, China ${ }^{2}$ Department of Neurosurgery, Lanzhou University Second Hospital, Lanzhou, China

${ }^{3}$ The Second Clinical Medical College, Lanzhou University, Lanzhou, China

Correspondence to

Professor Hao Chen;

ery_chenh@|zu.edu.cn

\section{ABSTRACT}

Introduction Venous thromboembolism (VTE) is a serious life-threatening complication in patients with gastric cancer. Abnormal coagulation function and tumour-related treatment may contribute to the occurrence of VTE. Many guidelines considered that surgical treatment would put patients with cancer at high risk of VTE, so positive prevention is needed. However, there are no studies that have systematically reviewed the postoperative risk and distribution of VTE in patients with gastric cancer. We thus conduct this systematic review to determine the risk of VTE in patients with gastric cancer undergoing surgery and provide some evidence for clinical decision-making. Methods and analysis Studies reporting the incidence of VTE after gastric cancer surgery will be included. Primary studies of randomised controlled trials, cohort studies, population-based surveys and cross-sectional studies are eligible for this review and only studies published in Chinese and English will be included. We will search the Medline, Embase, Web of Science, CBM, CNKI and Wanfang data from their inception to November 2019. Two reviewers will independently select studies and extract data. The quality of each included study will be assessed with tools corresponding to their study design. Meta-analysis will be used to pool the incidence data from included studies. Heterogeneity of the estimates across studies will be assessed, if necessary, a subgroup analysis will be performed to explore the source of heterogeneity. The Grades of Recommendation, Assessment, Development and Evaluation method is applied to assess the level of evidence obtained from this systematic review. Ethics and dissemination This proposed systematic review and meta-analysis is based on published data, and thus ethical approval is not required. The results of this review will be sought for publication.

PROSPERO registration number CRD42019144562

\section{INTRODUCTION}

Gastric cancer is one of the most common causes of cancer-related deaths in the world. ${ }^{12}$ Surgery is the main treatment for resectable gastric cancer. Venous thromboembolism (VTE), including deep vein thrombosis (DVT) and pulmonary embolism (PE), is a serious life-threatening complication in
Strengths and limitations of this study

- This review will determine the postoperative incidence of venous thromboembolism (VTE) in patients with gastric cancer.

- A subgroup analysis of certain patient characteristics (eg, patient race, gender and the surgery types patients received) makes it possible to identify the distribution of VTE incidence in gastric cancer surgery patients.

- There may be some heterogeneity across studies.

- Only studies published in Chinese and English will be included due to the language barrier, which may cause the loss of data from other languages.

patients with gastric cancer. Treatment of VTE imposes huge medical and economic costs and so it is crucial to prevent the occurrence of VTE.

Abnormal coagulation function and tumour-related treatment increase the risk of VTE in patients with tumour. Studies showed that the risk of VTE in patients with cancer increases about 50-fold compared with patients with non-cancer. ${ }^{3-9}$ Surgery is an independent risk factor for the occurrence of VTE, so theoretically, surgery for patients with cancer will have a higher risk of VTE. Therefore, both the National Comprehensive Cancer Network guidelines and the American Society of Clinical Oncology guidelines recommend routine preoperative use of anticoagulants to prevent thrombosis in patients with gastric cancer. ${ }^{1011}$ However, surgeons from Asian countries seldom use drugs to prevent thromboembolism, as the VTE is believed to be uncommon. ${ }^{12} 13$

At present, many research institutions have reported the incidence of VTE after gastric cancer surgery. ${ }^{14-16}$ However, the incidence of VTE varies greatly across different studies, which may be related to various factors. For 
example, VTE incidence in Asian countries was significantly lower than that in Western countries, which could be due to ethnic differences. Evaluating the incidence of VTE is important for guiding clinical decision-making. However, there is still a lack of comprehensive evaluations of the postoperative risk and distribution of VTE in patients with gastric cancer. Therefore, this study will use a systematic review method to determine the risk of VTE in patients with gastric cancer undergoing surgery, which will provide a reference for the need for routine preoperative use of pharmacologic thromboprophylaxis.

\section{METHODS AND ANALYSIS Objective}

To determine the incidence of VTE in patients undergoing surgical treatment of gastric cancer.

\section{Registration}

This protocol is being registered with PROSPERO (International Prospective Register of Systematic Reviews) (CRD42019144562)

\section{Eligibility criteria}

Participants

Patients with gastric cancer are eligible for this review. There are no restrictions on the basis of nationality or on the stage or grade of cancer. Patients with VTE at baseline will be excluded.

\section{Exposure}

The exposure of this review is surgical treatment. Patients with gastric cancer who receive surgery will be include. Surgical treatment refers to the standard procedure of resection for gastric cancer. Any type of surgery is valid, including laparoscopic and open surgery. The extent of surgical resection is not restricted. Patients who received VTE prophylaxis before or after surgery will be also included to reflect the influence of prophylaxis therapy. Patients who needed simultaneous surgery for other diseases will be excluded.

\section{Control}

Control is not necessary for this review, and no restriction for control will be made due to the aim of evaluating the overall VTE rates in patients with gastric cancer undergoing surgery. Studies without control will be also eligible for this review.

\section{Studies}

We will include reports on the incidence of VTE after gastric cancer surgery from randomised controlled trials, cohort studies, population-based surveys and cross-sectional studies. There is no limitation regarding the number of participants. Only studies published in Chinese and English will be included.

\section{Outcomes}

We will only consider studies whose primary outcomes include the VTE event. The studies should report the number of patients who developed a VTE event after gastric cancer surgery during the course of the study, with enough data to compute the VTE incidence after surgery. VTE events include symptomatic or incidentally detected DVT and PE. Any approach used to diagnose VTE was acceptable as long as a good diagnostic performance has been confirmed for this diagnosis tool, which may include clinical assessment, imaging, D-dimer testing and relevant diagnostic algorithms. Duration of follow-up has no restriction, but those without a description of follow-up length will be excluded. Studies occasionally reporting VTE as one of the adverse effects of surgery were not considered as these studies may selectively report only more severe cases or fail to strictly identify and classify thrombotic events.

\section{Information sources}

We will search the Medline, Embase, Web of Science, CBM (Chinese Biomedical Literature Database), CNKI (China National Knowledge Infrastructure) and Wanfang data databases from their inception to November 2019.

\section{Search strategy}

The search will be performed using a combination of subject terms with free-text terms. Search words will be adopted for each database and will mainly include the following: Thrombosis, Venous Thromboembolism, Pulmonary Embolism, PE, DVT, VTE, stomach neoplasms, stomach cancer, stomach tumour, gastric cancer, gastric tumour, Epidemiologic studies, Incidence in English and 血栓栓塞, 肺栓塞, 深静脉血栓, 血栓形成, 胃癌, 胃肿 瘤, 发生率, 流行 in Chinese. An example of the Medline search strategy is shown in table 1 . We will scan the reference lists of eligible studies and relevant review articles to identify any potentially eligible studies missed during our search. We will also search Google Scholar to identify any grey literature.

\section{Study records}

Data management

All the records retrieved from the databases are processed through EndNote X9. All extracted data are stored in a Microsoft Excel spreadsheet.

\section{Study selection and data collection process}

After eliminating duplicate articles, two reviewers will independently screen the titles and abstracts of all obtained articles, after which, the full texts of the potentially eligible articles will be retrieved. The same reviewers will then review the full texts of the selected articles. Any discrepancy will be resolved by face-to-face discussion or by the arbitration of a third reviewer. A data extraction form will be created to collect information from the studies. With regard to data of VTE incidence, we will categorise the results based on follow-up length, and those data of VTE reported at similar times will be grouped as a specified period of interest. Data from each arm in a multi-arms study will be extracted if all arms meet the above-mentioned eligibility criteria. Two reviewers will 


\begin{tabular}{|c|c|}
\hline 1 & $\begin{array}{l}\text { Thrombosis(Mesh) OR Thromboembolism(Mesh) } \\
\text { OR Venous Thromboembolism(Mesh) OR Venous } \\
\text { Thrombosis(Mesh) OR Pulmonary Embolism(Mesh) }\end{array}$ \\
\hline 2 & $\begin{array}{l}\text { thrombus }{ }^{\star}\left(\text { tiab) OR thrombotic }{ }^{\star}(\text { tiab) OR }\right. \\
\text { thrombolic*(tiab) OR thromboemboli*(tiab) OR } \\
\text { thrombos*(tiab) OR embol*(tiab) }\end{array}$ \\
\hline 3 & PE(tiab) OR DVT(tiab) OR VTE(tiab) \\
\hline 4 & (vein*(tiab) OR venous(tiab)) AND thromb*(tiab) \\
\hline 5 & \#1-4 OR \\
\hline 6 & 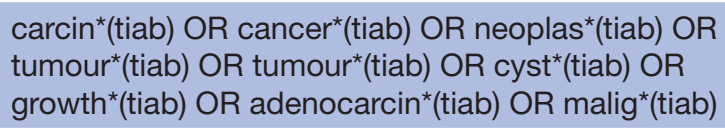 \\
\hline 7 & $\begin{array}{l}\text { Intestin*(tiab) OR Digest*(tiab) OR Gastr*(tiab) OR } \\
\text { gut(tiab) OR epigastr*(tiab) OR stomach*(tiab) }\end{array}$ \\
\hline 8 & \#6 AND \#7 \\
\hline 9 & $\begin{array}{l}\text { abdominal neoplasms(Mesh) OR intestinal } \\
\text { neoplasms(Mesh) OR stomach neoplasms(Mesh) }\end{array}$ \\
\hline 10 & \#8 OR \#9 \\
\hline 11 & $\begin{array}{l}\text { Epidemiologic studies(MeSh) OR Case-Control } \\
\text { Studies(MeSh) OR Cohort Studies(MeSh) OR } \\
\text { Incidence(MeSh) }\end{array}$ \\
\hline 12 & $\begin{array}{l}\text { case control(tiab) OR cohort study(tiab) OR } \\
\text { cohort studies(tiab) OR cohort analy^(tiab) } \\
\text { OR Follow-up study(tiab) OR Follow-up } \\
\text { studies(tiab) OR observational study(tiab) OR } \\
\text { observational studies(tiab) OR longitudinal(tiab) OR } \\
\text { retrospective(tiab) }\end{array}$ \\
\hline 13 & \#11 OR \#12 \\
\hline 14 & \#5 AND \#10 AND \#13 \\
\hline
\end{tabular}

independently extract the data from each included study, and a final check of the data will be conducted by a third researcher.

\section{Data items}

The data extracted include first author, publication year, continent, country, study design, number of participants, follow-up period, baseline, and specific characteristics of patients, type of surgery (laparoscopic or open surgery), incidence and type of VTE (PE or DVT of the lower or upper extremities) after surgery, presentation of VTE (symptomatic or incidentally detected), and use of thromboprophylaxis.

\section{Risk of bias in individual studies}

Two researchers will independently assess the quality of each included study using the tool of bias assessment for prevalence studies developed by Hoy et $a l^{17}$ and the Cochrane's bias assessment tool for randomised studies. ${ }^{18}$ Any discrepancies will be resolved by a third reviewer.

The assessment tool by Hoy et al examines the internal and external validity by taking into account study design, methodology and the presence of bias. Based on the scores assessed by this tool, we will rate each study as low risk, moderate risk and high risk. The Cochrane 'Risk of bias' assessment tool contains six domains as follows: selection bias (random sequence generation and allocation concealment), performance bias (blinding of participants and personnel), detection bias (blinding of outcome assessments), attrition bias (incomplete outcome data), reporting bias (selective reporting) and other bias (other sources of bias). We will classify risk of bias as 'low', 'high' or 'unclear' for all these domains. Risk of bias and quality scores will be summarised in a table.

\section{Data synthesis}

Estimates for the incidence of postoperative VTE will be pooled into a meta-analysis and displayed with $95 \%$ CIs. Study-specific incidence and SE estimates will be recalculated using crude numerators and denominators from the individual studies. An overall pooled estimate of the incidence will be calculated with a random effect model, ${ }^{19}$ using maximum likelihood estimation methods ${ }^{20}$ and modelling within-study variance as binomial. Heterogeneity will be assessed using Cochrane's $Q$ and will be quantified using the $\mathrm{I}^{2}$ statistics. Values of $25 \%, 50 \%$ and $75 \%$ for $\mathrm{I}^{2}$ will, respectively, represent low, medium and high heterogeneity. If substantial heterogeneity $(>50 \%)$ is observed, subgroup analysis using the following variables may be performed to explore the source of heterogeneity: study design (prospective vs retrospective); gender (male vs female); stage or grade of the cancer; type of surgery (laparoscopic vs open surgery); type of VTE; presentation of VTE. In addition, we will explore the influence of region, follow-up time and thromboprophylaxis use on VTE incidence. VTE incidence from different region (Asiatic and non-Asiatic), follow-up length (short period, long period or any other possible range), and modality of thromboprophylaxis (pre-surgical and post-surgical preventive measures) will be compared and tested for difference. All statistical tests will be calculated using the 'meta' package of the R statistical software (V.3.5.1, The R Foundation for statistical computing, Vienna, Austria). If the extracted data are not appropriate for pooling, narrative statistics will be performed.

\section{Meta-biases}

Publication bias will be explored graphically using a funnel plot and statistically using the Egger's test. ${ }^{21} \mathrm{p}<0.05$ is considered to indicate publication bias.

\section{Confidence in cumulative evidence}

The Grades of Recommendation, Assessment, Development and Evaluation method will be applied to assess the level of evidence obtained from this systematic review. ${ }^{22}$

\section{Methodology and reporting guidance for the review}

This review will be conducted strictly adhere to the Cochrane handbook and the Conducting Systematic Reviews and Meta-Analyses of Observational Studies of Etiology statements. ${ }^{23}$ The results of this review will be reported according to the Preferred Reporting Items for Systematic reviews and Meta-Analyses guidelines. ${ }^{25}$ We will include a flow diagram on how the studies were 
selected and a list of excluded studies and the reasons for exclusion.

\section{Patient and public involvement}

No patients or public will be involved in the study.

\section{DISCUSSION}

This systematic review will determine the incidence of VTE in patients who undergo surgery for gastric cancer. Several American and European guidelines recommend pharmacologic thromboprophylaxis for cancer surgery patients to prevent VTE. ${ }^{10} 1126$ However, many Asian surgeons do not follow these guidelines because they believe that the incidence of postoperative VTE is not high. ${ }^{12} 13$ Currently, there are many reports on the incidence of postoperative VTE in gastric cancer; however, a systematic review of the evidence is still lacking. The results of our review will indicate the risk and incidence distribution of VTE after gastric cancer surgery, which will provide a reference for the clinical decision regarding pharmacologic thromboprophylaxis.

There are also some possible limitations in our review. First, there may be some heterogeneity across studies as the study populations' baseline for each trial and the study design are heterogeneous. To explore the possible sources of heterogeneity, we will perform subgroup analysis. Second, only studies published in Chinese and English data will be included, to overcome the language barrier. Thus, we may lose data in other languages which may cause publication bias to some extent. Third, this study only focused on the overall VTE rates after surgery but did not estimate the increased magnitude of VTE risk due to surgery in patients with gastric cancer, nor the value of VTE prophylaxis for patients with gastric cancer undergoing surgery. We will perform further studies to address this question in the near future.

Contributors DFW and HC conceived the idea and planned the entire methods to undertake it. DFW and YY wrote the manuscript of this protocol. YY, PXT, DW and YJC designed the search strategy and planned the data extraction. All authors made contributions for conceiving this research project, and revised and approved the final version of the manuscript.

Funding This work will be supported by National Natural Science Foundation of China (81670594, 81470791, 81376597), Basic Research Innovation Group Project of Gansu Province (1606RJIA328), Scientific Research Project of Gansu Health Industry (GSWSKY2017-09), Fundamental Research Funds for the Central Universities (Izujbky-2016-k16, Izujbky-2017-79), Key Project of Science and Technology in Gansu province (19ZD2WA001), Talents Innovation and Entrepreneurship Program of Lanzhou City (2017-RC-62), Talent Staff Fund of the Lanzhou University Second Hospital (ynyjrckyzx2015-1-01), and Cuiying Scientific and Technological Innovation Program of Lanzhou University Second Hospital (CY2017-ZD01)

\section{Competing interests None declared.}

Patient consent for publication Not required.

Ethics approval This proposed systematic review and meta-analysis is based on published data, and thus ethical approval is not required. The results of this review will be sought for publication.

Provenance and peer review Not commissioned; externally peer reviewed.

Open access This is an open access article distributed in accordance with the Creative Commons Attribution Non Commercial (CC BY-NC 4.0) license, which permits others to distribute, remix, adapt, build upon this work non-commercially, and license their derivative works on different terms, provided the original work is properly cited, appropriate credit is given, any changes made indicated, and the use is non-commercial. See: http://creativecommons.org/licenses/by-nc/4.0/.

ORCID iD

Yang Yu http://orcid.org/0000-0002-2409-3681

\section{REFERENCES}

1 Bray F, Ferlay J, Soerjomataram I, et al. Global cancer statistics 2018: GLOBOCAN estimates of incidence and mortality worldwide for 36 cancers in 185 countries. CA Cancer J Clin 2018;68:394-424.

2 Ferlay J, Soerjomataram I, Dikshit R, et al. Cancer incidence and mortality worldwide: sources, methods and major patterns in GLOBOCAN 2012. Int J Cancer 2015;136:E359-86.

3 Barni S, Labianca R, Agnelli G, et al. Chemotherapy-Associated thromboembolic risk in cancer outpatients and effect of nadroparin thromboprophylaxis: results of a retrospective analysis of the PROTECHT study. J Transl Med 2011;9:179.

4 Cohen AT, Katholing A, Rietbrock S, et al. Epidemiology of first and recurrent venous thromboembolism in patients with active cancer. $A$ population-based cohort study. Thromb Haemost 2017;117:57-65.

5 Khorana AA, Connolly GC. Assessing risk of venous thromboembolism in the patient with cancer. J Clin Oncol 2009;27:4839-47.

6 Khorana AA, Francis CW, Culakova E, et al. Risk factors for chemotherapy-associated venous thromboembolism in a prospective observational study. Cancer 2005;104:2822-9.

7 Khorana AA, Kuderer NM, Culakova E, et al. Development and validation of a predictive model for chemotherapy-associated thrombosis. Blood 2008;111:4902-7.

8 Moore RA, Adel N, Riedel E, et al. High incidence of thromboembolic events in patients treated with cisplatin-based chemotherapy: a large retrospective analysis. JCO 2011;29:3466-73.

9 Qu C-Y, Zheng Y, Zhou M, et al. Value of bevacizumab in treatment of colorectal cancer: a meta-analysis. World J Gastroenterol 2015;21:5072-80.

10 Lyman GH, Bohlke K, Falanga A, et al. Venous thromboembolism prophylaxis and treatment in patients with cancer: American Society of clinical oncology clinical practice guideline update. J Oncol Pract 2015;11:e442-4.

11 Streiff MB, Holmstrom B, Ashrani A, et al. Cancer-Associated venous thromboembolic disease, version 1.2015. J Natl Compr Canc Netw 2015;13:1079-95.

12 Liew NC, Chang YH, Choi G, et al. Asian venous thromboembolism guidelines: prevention of venous thromboembolism. Int Angiol 2012;31:501-16.

13 Wang K-L, Yap ES, Goto S, et al. The diagnosis and treatment of venous thromboembolism in Asian patients. Thromb J 2018;16:4.

$14 \mathrm{Kim}$ JW, Chun EJ, Choi SI, et al. A prospective study on the incidence of postoperative venous thromboembolism in Korean gastric cancer patients: an inquiry into the application of Western guidelines to Asian cancer patients. PLoS One 2013;8:e61968.

15 Kimura Y, Oki E, Ando K, et al. Incidence of venous thromboembolism following laparoscopic surgery for gastrointestinal cancer: a single-center, prospective cohort study. World J Surg 2016;40:309-14.

16 Wada T, Fujiwara H, Morita $\mathrm{S}$, et al. Incidence of and risk factors for preoperative deep venous thrombosis in patients undergoing gastric cancer surgery. Gastric Cancer 2017;20:872-7.

17 Hoy D, Brooks P, Woolf A, et al. Assessing risk of bias in prevalence studies: modification of an existing tool and evidence of interrater agreement. J Clin Epidemiol 2012;65:934-9.

18 Higgins JPT, Altman DG, Gøtzsche PC, et al. The Cochrane collaboration's tool for assessing risk of bias in randomised trials. BMJ 2011;343:d5928.

19 Borenstein M, Hedges LV, Higgins JPT, et al. A basic introduction to fixed-effect and random-effects models for meta-analysis. Res Synth Methods 2010;1:97-111.

20 Hamza TH, van Houwelingen HC, Stijnen T. The binomial distribution of meta-analysis was preferred to model within-study variability. $J$ Clin Epidemiol 2008;61:41-51.

21 Duval S, Tweedie R. Trim and fill: a simple funnel-plot-based method of testing and adjusting for publication bias in meta-analysis. Biometrics 2000;56:455-63.

22 Iorio A, Spencer FA, Falavigna M, et al. Use of grade for assessment of evidence about prognosis: rating confidence in estimates of event rates in broad categories of patients. BMJ 2015;350:h870. 
23 Dekkers OM, Vandenbroucke JP, Cevallos M, et al. COSMOS-E: guidance on conducting systematic reviews and meta-analyses of observational studies of etiology. PLoS Med 2019;16:e1002742.

24 Higgins JP, Green S. Cochrane Handbook for systematic reviews of interventions. John Wiley \& Sons, 2011.
25 Moher D, Liberati A, Tetzlaff J, et al. Preferred reporting items for systematic reviews and meta-analyses: the PRISMA statement. BMJ 2009;339:b2535.

26 Mandalà M, Falanga $A$, Roila F, et al. Management of venous thromboembolism (VTe) in cancer patients: ESMO clinical practice guidelines. Ann Oncol 2011;22 Suppl 6:vi85-92. 